\title{
Principais indicações de transplante penetrante de córnea em um serviço de referência no interior de São Paulo (Sorocaba - SP, Brasil)
}

\author{
Major indications for corneal penetrating keratoplasty at a reference service in \\ São Paulo state (Sorocaba - SP, Brazil)
}

\author{
Miǵuel José Calix Netto ${ }^{1}$ \\ Eduardo Della Giustina ${ }^{2}$ \\ Gabriel Zatti Ramos ${ }^{3}$ \\ Rodrigo Favoretto Cañas Peccini ${ }^{4}$ \\ MarceloSobrinho ${ }^{5}$ \\ Luciene Barbosa de Souza ${ }^{6}$
}

Trabalho realizado no Setor de Córnea do Hospital Oftalmológico de Sorocaba.

Residente do terceiro ano do Hospital Oftalmológico de Sorocaba - HOS - Sorocaba (SP) - Brasil.

Residente do terceiro ano do HOS - Sorocaba (SP) Brasil.

Residente do segundo ano do HOS - Sorocaba (SP) Brasil.

Residente do segundo ano do HOS - Sorocaba (SP) Brasil.

${ }_{5}^{5}$ Preceptor do setor de lentes de contato do HOS - Sorocaba (SP) - Brasil.

${ }^{6}$ Coordenadora do Serviço de Residência Médica do HOS - Sorocaba (SP) - Brasil.

Endereço para correspondência: Miguel José Calix Netto. Rua Vicência Faria Versage, 75 - Apto. 42 Sorocaba (SP) CEP 18031-080

E-mail:vandotte@hotmail.com

Recebido para publicação em 29.05.2005

Última versão recebida em 25.01.2006

Aprovação em 12.02.2006

Nota Editorial: Depois de concluída a análise do artigo sob sigilo editorial e com a anuência do Dr. Renato Ambrósio Jr. sobre a divulgação de seu nome como revisor, agradecemos sua participação neste processo.

\begin{tabular}{|l|}
\hline RESUMO \\
\hline Objetivos: Identificar as principais indicações de transplante penetrante \\
de córnea e fornecer o perfil demográfico e epidemiológico dos pacientes \\
encaminhados ao Projeto de triagem de transplante do Hospital Oftalmo- \\
lógico de Sorocaba. Métodos: Foram analisados retrospectivamente os \\
prontuários dos pacientes, no período de junho a dezembro de 2003, que \\
foram encaminhados para o Projeto Transplante de Córnea em nosso \\
serviço. Resultados: Dos 171 pacientes estudados, 102 (59,6\%) pacientes \\
eram do sexo masculino, 69 (40,3\%) do sexo feminino; a média de idade \\
foi de 37 anos, 106 (49\%) possuíram indicação de transplante de córnea. \\
Procedência dos pacientes encaminhados ao projeto transplante: São \\
Paulo (68\%), Minas Gerais (6\%), Paraná (6\%), Rio de Janeiro (5\%), \\
Maranhão (3\%) e outros (12\%). As principais indicações de transplante \\
de córnea em nosso estudo foram: ceratocone 65\%, ceratopatia bolhosa \\
do pseudofácico 21\%, leucoma corneano 10\%, distrofia de Fuchs 1,9\%, \\
distrofia lattice 0,9\% e síndrome de Steven Johnson 0,9\%. Conclusão: \\
Muitos pacientes encaminhados para transplante em nosso serviço não \\
apresentavam indicação para o mesmo, fato que demonstra o desconhe- \\
cimento dos oftalmologistas gerais sobre as indicações reais do transplan- \\
te de córnea. Quanto às principais doenças indicadas para transplante o \\
ceratocone predominou, seguido da ceratopatia bolhosa do pseudofácico.
\end{tabular}

Descritores: Transplante de córnea; Ceratoplastia penetrante/epidemiologia; Ceratocone; Doenças da córnea/epidemiologia; Hospitais especializados; Estudos retrospectivos

\section{INTRODUÇÃO}

O transplante de córnea vem sendo praticado cada vez mais por oftalmologistas em todo Brasil. Esse aumento do número de transplantes ocorre devido à córnea ser um órgão privilegiado imunologicamente com menor risco de rejeição em comparação com outros órgãos, pela crescente conscientização da população para doação de órgãos e pelo surgimento de novos banco de olhos no Brasil ${ }^{(1-2)}$.

O Hospital Oftalmológico de Sorocaba (SP), fundado há oito anos, na cidade de Sorocaba é referência atualmente no País, como centro em transplantes de córnea. É feito um trabalho perante a comunidade incentivando a doação de córneas, gerando um número aproximado de 130 transplantes ao mês, com uma fila de espera de aproximadamente seis meses*.

*Dados coletados do banco de dados do Banco de Olhos de Sorocaba no ano de 2004. 
Existe um ambulatório no setor de córnea que realiza uma triagem dos pacientes para transplante, chamado "Projeto Transplante". Esses pacientes são encaminhados das mais diferentes regiões do País, com laudos de seus respectivos médicos oftalmologistas para serem submetidos a uma avaliação cirúrgica dando entrada pela primeira vez em nosso serviço para uma avaliação de transplante de córnea. Esse ambulatório é necessário ao nosso serviço, pois os encaminhamentos dos colegas oftalmologistas muitas vezes não condizem com as indicações corretas para o transplante de córnea.

O objetivo deste estudo foi identificar as principais indicações de transplante de córnea dos pacientes encaminhados ao nosso serviço, fornecendo também um perfil demográfico e epidemiológico.

\section{MÉTODOS}

Foram analisados retrospectivamente os prontuários dos pacientes que foram encaminhados para o Projeto Transplante no ambulatório de córnea e doenças externas do nosso serviço, no período de junho a dezembro de 2003. Este estudo teve a aprovação do comitê de ética em pesquisa do Hospital Oftalmológico de Sorocaba.

Os pacientes durante o Projeto Transplante, realizavam uma consulta oftalmológica completa padrão: com idade, sexo, raça, procedência, naturalidade, história oftalmológica e sistêmica, exame da acuidade visual Snellen, pressão ocular, exame biomicroscópico do segmento anterior e exame fundoscópico. Essa avaliação foi realizada pelos residentes e estagiários de córnea no hospital referido, sob a supervisão dos orientadores em córnea.

Se necessário, os pacientes eram encaminhados para teste com lente de contato, a fim de obter a melhor acuidade visual corrigida. Após realizar todos os exames complementares necessários, era decidido na mesma consulta se o paciente possuía indicação para transplante. Com essa avaliação, o paciente era orientado sobre o procedimento cirúrgico do transplante de córnea, explicando ao mesmo e a seus acompanhantes, as complicações intra e pós-operatórias e da necessidade de seguimento regular. Caso o paciente com indicação de transplante de córnea aceitasse os termos propostos, o mesmo era inscrito na fila de transplante do hospital.

Foram analisadas variáveis baseadas na ficha do projeto, como por exemplo: número total de pacientes e olhos, média de idade dos pacientes, sexo, procedência por estado, acuidade visual sem correção, número de indicação de transplante de córnea e as doenças dos olhos estudados.

\section{RESULTADOS}

Foram analisados durante o período de junho a dezembro de 2003, os prontuários de 171 pacientes, com o total de 217 olhos estudados. Quanto ao sexo, 102 pacientes eram do sexo masculino $(59,6 \%)$, ao passo que 69 pacientes eram do sexo feminino (40,3\%). A média de idade foi de 37 anos (variando de 3 a 83 anos). A procedência dos pacientes por estados brasileiros está sumarizada no gráfico 1 .

Dos olhos estudados 49\% (106) tiveram indicação de transplante de córnea; $18,4 \%$ (40) foram indicados ao uso de lentes de contato e $32,7 \%$ (71) dos olhos não possuíam indicação de transplante e nem indicação do uso de lentes de contato (ambliopia, visão com e sem percepção de luz, glaucoma, descolamento de retina, cicatriz macular, pacientes que simulavam a acuidade visual e deficientes mentais).

As principais doenças encaminhadas ao projeto, por outros oftalmologistas do país estão dispostas no gráfico 2.

As principais indicações de transplante de córnea feitas no nosso serviço encontram-se na tabela 1 .

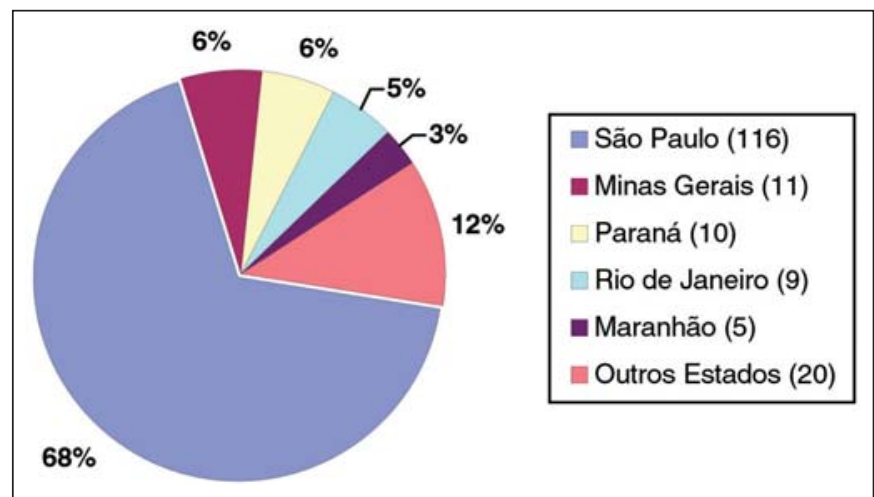

Gráfico 1 - Procedência dos pacientes por estados brasileiros
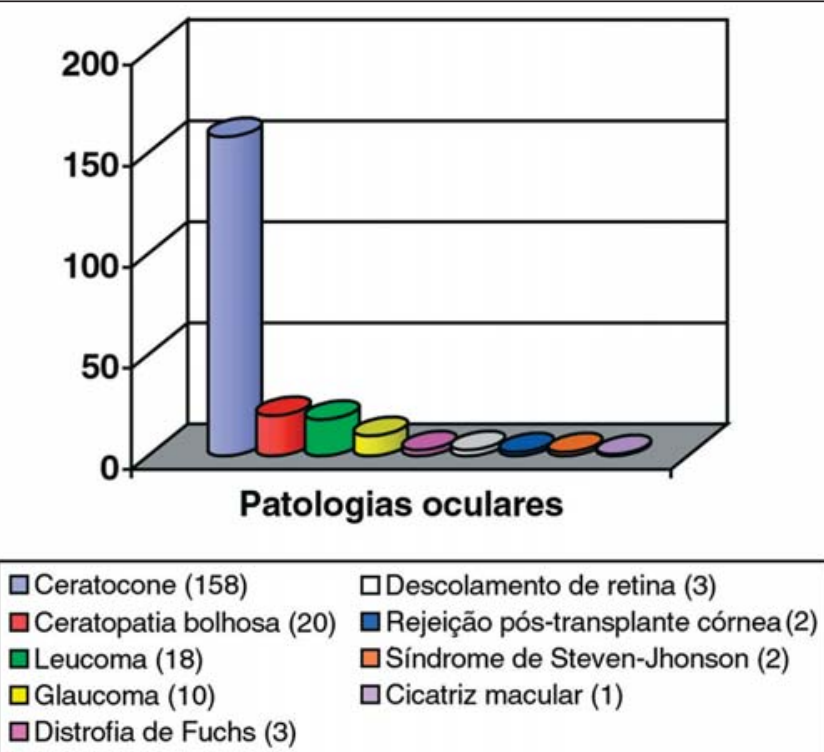

Gráfico 2 - Doenças encaminhadas para avaliação de transplante de córnea por número de olhos 


\section{DISCUSSÃO}

Em nosso estudo, foram avaliadas as indicações de transplante penetrante de córnea por um período de sete meses (junho a dezembro de 2003), num hospital de referência no estado de São Paulo.

O transplante de córnea como transplante de órgão humano é seguro e de prática comum ${ }^{(2)}$. Há uma intensa propaganda nos meios de comunicação sobre o transplante de córnea, muitas vezes sendo tomado como solução "milagrosa" para doenças que às vezes não tem indicação para tal procedimento. Por essa razão e pelo relativo desconhecimento dos oftalmologistas gerais, é necessário fazer uma triagem para os pacientes encaminhados pelos nossos colegas, reavaliando suas indicações.

Encontramos menos de $50 \%$ de indicações corretas para o transplante de córnea nos pacientes encaminhados ao nosso ambulatório feitos por oftalmologista das mais variadas regiões do país o que era comprovado pela guia de encaminhamento assinada. Algumas doenças eram de segmento posterior como cicatriz macular e descolamento de retina, evidenciando a pouca compreensão dos colegas sobre a ceratoplastia penetrante a qual apenas substitui a córnea pouco funcional por outra opticamente saudável. Em outros casos, a adaptação de lentes corretivas como óculos e lentes de contato resolveram a baixa acuidade visual em 18,4\% dos pacientes encaminhados, mostrando como é importante encaminhar os pacientes com alterações corneanas para um teste com lentes de contato a um profissional capacitado para tal. Isso permite uma redução nas indicações de transplante de córnea e uma melhor qualidade de vida para o paciente.

\begin{tabular}{|c|c|c|}
\hline & Número de pacientes & Porcentagem \\
\hline Ceratocone & 69 & $65,0 \%$ \\
\hline $\begin{array}{l}\text { Ceratopatia bolhosa } \\
\text { do pseudofácico }\end{array}$ & 22 & $21,0 \%$ \\
\hline Leucoma & 11 & $10,0 \%$ \\
\hline Distrofia de Fuchs & 2 & $1,9 \%$ \\
\hline Distrofia lattice & 1 & $0,9 \%$ \\
\hline Stevens-Johnson & 1 & $0,9 \%$ \\
\hline
\end{tabular}

As principais indicações de transplante penetrante de córnea da literatura internacional comparada às indicações feitas em nosso ambulatório, constam na tabela 2.

Para nós, o ceratocone foi a principal indicação de transplante penetrante de córnea representando $65 \%$ do total. Esse dado se diferencia um pouco da literatura pesquisada em que o ceratocone predominou entre a segunda e terceira indicações de transplante variando de 11,4 a $20,7 \%$ do total ${ }^{(1-6)}$. A razão dessa diferença pode ser explicada por variações no sucesso da adaptação de lente de contato ou secundário a fatores socioeconômicos e climáticos que puderam influenciar na tolerância e uso. Além disso, os pacientes são enviados ao nosso serviço geralmente após teste com lente de contato em sua cidade de origem, ter sido feito sem sucesso ou que não tenham melhorado sua acuidade visual, já com a indicação de transplante de córnea feito pelo seu oftalmologista.

Em nosso serviço não é feito de rotina anel intra-estromal como alternativa para tratamento do ceratocone, com isso não entrou na estatística do Projeto Transplante no período em estudo. Recebemos pacientes com implantes de anel intraestromal provenientes de outros serviços em que não houve melhora significativa da acuidade visual encaminhados como indicação de transplante de córnea, passando nesse caso pela triagem do projeto.

A média de idade dos pacientes com indicação de transplante penetrante de córnea em nosso estudo foi de 37 anos, o que é compatível com a média de idade de indicação de transplante para ceratocone que é de 34,43 anos $^{(3)}$; justificando a predominância desta indicação em nosso serviço.

A segunda maior indicação de transplante em nosso estudo foi a ceratopatia bolhosa representando $21 \%$ do total, o que está em acordo com a maioria dos estudos pesquisados em que variou entre 18,4 a $39,0 \%{ }^{(1-6)}$.

O transplante penetrante de córnea é muito comum e a incidência de rejeição está em torno de $30 \%{ }^{(2)}$. A falência do transplante de córnea não foi observada nas indicações do nosso Projeto Transplante, diferenciando da literatura, em que sempre permanecia entre as primeiras indicações de transplante ${ }^{(3)}$. Essa ausência de re-transplantes como indicação em nosso estudo pode ser explicada, devido a grande maioria dos pacientes encaminhados para o Projeto Transplante serem casos novos e sem cirurgia ocular prévia. No ambulatório de

\begin{tabular}{|c|c|c|c|c|c|c|c|}
\hline & $\begin{array}{c}\text { Kendall } \\
\text { et al. } .^{(1)}, 2000\end{array}$ & $\begin{array}{c}\text { Kenji } \\
\text { et al. } .^{(2)}, 1999\end{array}$ & $\begin{array}{c}\text { Maeno } \\
\text { et } \mathrm{al}^{(3)}, 2000\end{array}$ & $\begin{array}{c}\text { Niraj } \\
\text { et al. } .^{(4)}, 2000\end{array}$ & $\begin{array}{c}\text { Smith } \\
\text { et al. } .^{(5)}, 1980\end{array}$ & $\begin{array}{c}\text { Robin } \\
\text { et al.(6), } 1986\end{array}$ & $\begin{array}{l}\text { Indicações do } \\
\text { presente estudo }\end{array}$ \\
\hline $\begin{array}{l}\text { Ceratopatia bolhosa do } \\
\text { pseudofácico e afácico }\end{array}$ & $39,0 \%$ & $23,7 \%$ & $18,94 \%$ & $31,9 \%$ & $18,4 \%$ & $28,4 \%$ & $21,0 \%$ \\
\hline Distrofia de Fuchs & $23,2 \%$ & $6,6 \%$ & $9,56 \%$ & $18,2 \%$ & $9,8 \%$ & $9,1 \%$ & $1,9 \%$ \\
\hline Ceratocone & $11,4 \%$ & $20,7 \%$ & $16,04 \%$ & $15,3 \%$ & $12,7 \%$ & & $65,0 \%$ \\
\hline Cicatriz corneana & $11,2 \%$ & $29,8 \%$ & $6,16 \%$ & $1,5 \%$ & & $9,3 \%$ & $10,0 \%$ \\
\hline Falência do transplante & $8,9 \%$ & $17,2 \%$ & $18,01 \%$ & $16,0 \%$ & $15,4 \%$ & $15,4 \%$ & \\
\hline Outras indicações & $7,0 \%$ & $2,0 \%$ & $31,29 \%$ & $17,1 \%$ & $43,7 \%$ & $37,8 \%$ & $1,8 \%$ \\
\hline
\end{tabular}


córnea do nosso serviço, onde os casos de transplantes, após as cirurgias indicadas pelo projeto serem realizadas, são acompanhados, se houvesse rejeição já eram automaticamente reinscritos na fila de transplante, sendo considerados como priorização não sendo re-encaminhados novamente ao Projeto, justificando assim a baixa freqüência de falência do transplante de córnea como indicação em nosso estudo.

Apesar dos avanços na imunossupressão ocular, na terapia antiglaucomatosa, antibiótica, na técnica de transplante e cuidados no pós-operatório, a falência do botão do transplante ainda se mantém como indicação comum de transplante penetrante $^{(1-4)}$ e sua incidência está em torno de 8,90 a 18,01\%(1-6).

Alguns autores relataram o aumento da porcentagem de transplantes penetrantes repetidos, com o re-transplante se tornando a indicação mais comum. Esse estudo revelou que o re-transplante foi a segunda indicação mais comum com $18 \%$ dos casos, seguido da ceratopatia bolhosa do pseudofácico que representou $26 \%$ dos $\operatorname{casos}^{(7)}$. Sharif e Casey, relataram que $41 \%$ dos transplantes realizados entre 1971 e 1990 foram por falência do transplante anterior, o que representou a maior porcentagem achada em todos os estudos de revisão ${ }^{(8)}$. Flower et al., comparou dados de re-transplante, mostrando que representou $21 \%$ do total de transplantes realizados de 1989 a $1993^{(9)}$. Em nosso estudo, como já discutimos acima, não observamos como indicação de transplante de córnea a falência do botão doador.

Futuramente as indicações de transplante de córnea poderão sofrer a influência de diversos fatores, entre eles a tendência de aumento de doadores devido a maior divulgação e conscientização da população, aumento do número de banco de olhos no Brasil, melhora da técnica e equipamentos cirúrgicos bem como os meios de preservação.

Isso encorajará muitos cirurgiões a transplantar um maior número de pacientes inclusive aqueles de pior prognóstico. Se essa tendência se firmar, o re-transplante poderá se tornar a primeira indicação de "novo" transplante penetrante de córnea como já foi citado por alguns autores ${ }^{(8)}$.

\section{CONCLUSÃO}

Muitos pacientes encaminhados para transplante em nosso serviço não apresentavam indicação para o mesmo, fato que demonstra o desconhecimento dos oftalmologistas gerais sobre as indicações reais do transplante de córnea. Quanto às principais doenças indicadas para transplante o ceratocone predominou, seguido da bolhosa do pseudofácico.

\section{ABSTRACT}

Purpose: To identify the main indications for corneal transplantation, analyze the results and provide demographic and epidemiological profile of patients referred to the Corneal Transplantation Project. Methods: A retrospective analysis, from June to December 2003, of patients referred to the Corneal Transplantation Project. Results: Total of 171 patients. One hundred and two (59.6\%) patients were male and 69 $(40.3 \%)$ female; mean age was 37 years. One hundred and six (49\%) patients had indication for corneal transplantation. The origins of patients were: São Paulo (68\%), Minas Gerais (6\%), Paraná (6\%), Rio de Janeiro (5\%), Maranhão (3\%) and others (12\%). The main indications: keratoconus $65 \%$, pseudophakic bullous keratopathy $21 \%$, corneal scarring $10 \%$, Fuchs dystrophy $1.9 \%$, lattice dystrophy $0.9 \%$ and Stevens-Johnson syndrome $0.9 \%$. Conclusion: Many patients referred to corneal transplantation had no indication for such procedure. This fact demonstrates the poor knowledge of general ophthalmologists about the real indications of penetrating keratoplasty. Keratoconus was the major indication followed by pseudophakic bullous keratopathy.

Keywords: Corneal transplantation; Keratoplasty, penetrating/epidemiology; Keratoconus; Corneal diseases/epidemiology; Hospitals, special; Retrospective studies

\section{REFERÊNCIAS}

1. Dobbins KR, Price FW Jr, Whitson WE. Trends in the indications for penetrating keratoplasty in the midwestern United States. Cornea. 2000;19(6):813-6.

2. Inoue K, Amano S, Oshika T, Sawa M, Tsuru T. A 10-year review of penetrating keratoplasty. Jpn J Ophthalmol. 2000;44(2):139-45.

3. Maeno A, Naor J, Lee HM, Hunter WS, Rootman DS. Three decades of corneal transplantation: indications and patient characteristics. Cornea. 2000;19(1):7-11.

4. Patel NP, Kim T, Rapuano CJ, Cohen EJ, Laibson PR. Indications for and outcomes of repeat penetrating keratoplasty, 1989-1995. Ophthalmology. 2000; 107(4):719-24.

5. Smith RE, McDonald HR, Nesburn AB, Minckler DS. Penetrating keratoplasty: changing indications, 1947 to 1978. Arch Ophthalmol. 1980;98(7):1226-9.

6. Robin JB, Gindi JJ, Koh K, Schanzlin DJ, Rao NA, York KK, et al. An update of the indications for penetrating keratoplasty. 1979 through 1983. Arch Ophthalmol. 1986;104(1):87-9.

7. Lois N, Kowal VO, Cohen EJ, Rapuano CJ, Gault JA, Raber IM, et al. Indications for penetrating keratoplasty and associated procedures, 1989-1995. Cornea. 1997;16(6):623-9.

8. Sharif KW, Casey TA. Changing indications for penetrating keratoplasty, 19711990. Eye. 1993;7(Pt 4):485-8.

9. Flowers CW, Chang KY, McLeod SD, Irvine JA, McDonnell PJ, Rao N, et al. Changing indications for penetrating keratoplasty, 1989-1993. Cornea. 1995;14(6):583-8. Comment in: Cornea. 1997;16(1):120-1. 